\title{
Short-term effectiveness and safety of an anatomy-conforming hybrid surgery protocol for thoracoabdominal aortic aneurysms
}

\author{
Yuejin $\mathrm{Li}^{1} \wedge$, Le Xiao ${ }^{1}$, Peng Zhang ${ }^{1}$, Jian Zhang ${ }^{1}$, Shikui Guo ${ }^{1}$, Rougang $\mathrm{Li}^{1}$, Yan Wei ${ }^{2}$, Kunmei Gong ${ }^{1} \wedge$ \\ ${ }^{1}$ Department of General Surgery, the First People's Hospital of Yunnan Province \& the Affiliated Hospital of Kunming University of Science and \\ Technology, Kunming, China; ${ }^{2}$ Department of General Surgery, Kunming University of Science and Technology School of Medicine \& Affiliated \\ Hospital and the First People's Hospital of Yunnan Province, Kunming, China \\ Contributions: (I) Conception and design: K Gong, Y Li; (II) Administrative support: K Gong; (III) Provision of study materials or patients: L Xiao, P \\ Zhang, J Zhang, S Guo, R Li; (IV) Collection and assembly of data: Y Wei; (V) Data analysis and interpretation: Y Li; (VI) Manuscript writing: All \\ authors; (VII) Final approval of manuscript: All authors. \\ Correspondence to: Kunmei Gong. Department of General Surgery, the First People's Hospital of Yunnan Province \& the Affiliated Hospital of \\ Kunming University of Science and Technology, Kunming, China. Email: kunhuagongkunmei@163.com.
}

\begin{abstract}
Background: Thoracoabdominal aortic aneurysms (TAAAs) are one of the most complex aortic aneurysms to treat. Traditional open surgery for TAAAs is highly invasive and total endovascular treatment still has many limitations. Hybrid surgery offers a viable option for TAAA patients, but currently it often alters the original hemodynamics. Therefore, we have developed and applied a hybrid surgery protocol for anatomically suitable TAAA patients. Here we analyzed the short-term outcomes of these patients and evaluated the effectiveness and safety of this procedure.
\end{abstract}

Methods: A new method was used to perform the required artificial revascularization of the abdominal aorta and its branches. A new visceral debranching and reconstruction approach was used to complete an anatomy-conforming open surgery. Finally, a new vascular access was used for the endovascular operation to complete the implantation of stent-grafts. The clinical data of 10 patients with TAAAs treated at our center with the anatomy-conforming hybrid technique during the period from May 2018 to June 2020 was retrospectively analyzed. The intraoperative and 30-day postoperative key indicators, including survival rates and complications, were analyzed.

Results: The hybrid surgery was smoothly performed in all 10 patients. One patient died from non-aortic causes (sepsis/infectious toxic shock) 30 days after surgery, yielding a case-fatality rate of $10.0 \%(1 / 10)$. Two patients (2/10) experienced serious complications within 30 days postoperatively, including multiorgan failure due to infection in one patient and lower limb ischemia in another patient who went on to develop permanent paraplegia. No endoleak after stent implantation was noted and the blood supply of each reconstructed visceral artery was good.

Conclusions: Based on the short-term outcomes, the anatomy-conforming hybrid surgical protocol is safe and effective in treating TAAA patients.

Keywords: Thoracoabdominal aortic aneurysm (TAAA); anatomical features; hybrid surgery

Submitted May 15, 2021. Accepted for publication Jul 26, 2021.

doi: 10.21037/apm-21-1569

View this article at: https://dx.doi.org/10.21037/apm-21-1569

^ ORCID: Yuejin Li, 0000-0002-9978-0064; Kunmei Gong, 0000-0003-0823-5165. 


\section{Introduction}

Thoracoabdominal aortic aneurysms (TAAAs) are aneurysms involving the celiac trunk, superior mesenteric artery, and both renal arteries, the incidence of which is 5.9/100,000 per year (1). Typically, TAAAs are grouped into four types (I-IV) using the Crawford classification. When a TAAA is accompanied by an aortic dissection or develops on the basis of a dissection, more complex aortic dissecting aneurysms (ADAs) may occur. These include: (I) suprarenal or renal abdominal ADAs; (II) aortic dissection accompanied by aortic aneurysms (thoracic and/or abdominal); and (III) development of TAAA following a surgery [e.g., total arch replacement + stented elephant trunk technique or thoracic endovascular aortic repair (TEVAR)] for a thoracic aortic dissection. These complex thoracoabdominal aortic lesions are large, involving many organs and affecting a wide range of functions.

In 1998, Quiñones-Baldrich et al. described for the first the combination of infrarenal aortic replacement with stent implantation for the treatment to thoracoabdominal aortic pathology (2). This combined endovascular and surgical approach reduced morbidity and mortality with good mid-term results $(2,3)$. Compared with open surgery, the hybrid approach has smaller surgical incisions and shorter operative time, without the need for thoracotomy, single-lung ventilation, high aortic blockade, and/or extracorporeal circulation; theoretically, it reduces the incidence of pulmonary complications and spinal cord ischemia (4). It also partially solves the problems of speed, feasibility and cost in endovascular treatment. The hybrid surgery combines the advantages of open surgery and endovascular repair (5). It offers a novel treatment option for patients who cannot tolerate traditional open surgery and/or whose lesions are too extensive and anatomically complex to be treated with endovascular repair.

When a hybrid repair of a TAAA is performed for visceral artery reconstruction, the end-lateral anastomosis between an artificial trunk and the abdominal aorta or iliac artery is often used as an inflow tract, followed by the end-lateral anastomoses between artificial vessel branches and visceral arteries. This approach is a non-anatomical reconstruction method as the hemodynamics of the blood flow to visceral arteries is altered (6). Thus, a new hybrid surgical protocol for the treatment of TAAA has been developed in our center, with an attempt to reconstruct the visceral arteries in an anatomy-conforming manner. This article summarizes the short-term outcomes and complications of this procedure, thus providing a preliminary assessment of its effectiveness and safety. We present the following article in accordance with the STROBE reporting checklist (available at https://dx.doi.org/10.21037/apm-21-1569).

\section{Methods}

\section{General data}

It added up to 10 patients with TAAAs who were treated at our center with a hybrid technique consisting of infrarenal aortic replacement and stent implantation during the period from May 2018 to June 2020. The clinical data of all these 10 patients were retrospectively analyzed. Eight of these 10 patients were male and two were female, aged $45.3 \pm 12.5$ years (range, 27-62 years). The commodities included hypertension $(n=6)$, diabetes $(n=1)$, cardiac insufficiency $(n=5)$, and renal insufficiency $(n=2)$. All patients underwent preoperative computed tomographic angiography (CTA) from the aortic arch to the root of the bilateral superficial femoral arteries and were diagnosed with TAAA (with or without aortic dissection). More specifically, there were four cases of distal dissecting aneurysm following TEVAR for type B aortic dissection (pending aneurysm rupture in one case), three cases of distal dissecting aneurysm following open thoracic aortic arch replacement (pending aneurysm rupture in one case), and three cases of TAAA [type IV rupture in one case and type I infrarenal abdominal aortic stenosis (near occlusion) in one case]. The surgeries were simultaneous in 9 cases and staged (stage 1: replacement of the infrarenal aorta + debranching; and stage 2: placement of a stent graft in the descending thoracic aorta) in one case. The maximum diameter of the aneurysm was $6.19 \pm 1.72 \mathrm{~cm}$ (range, 4.6 to $9.8 \mathrm{~cm}$ ). The clinical data and anatomic features of these patients are summarized in Table 1. All the patients and their families signed informed consent forms. The study was approved by the Research Ethics Sub-Committee of the Medical Ethics Committee of the First People's Hospital of Yunnan Province (approval number: KHLL2020-KY049). All procedures performed in this study involving human participants were in accordance with the Declaration of Helsinki (as revised in 2013).

\section{Surgical techniques}

\section{A new method for reconstructing the abdominal aorta and its branches}

A HEMASHIELD Platinum Woven Double Velour Vascular Graft (hereafter referred as "artificial four-branch trunk") was used for the reconstruction of the abdominal aorta and its branches. The graft was re-cut, spliced, and 
Table 1 General data, anatomic features, and intraoperative outcomes of ten TAAA patients

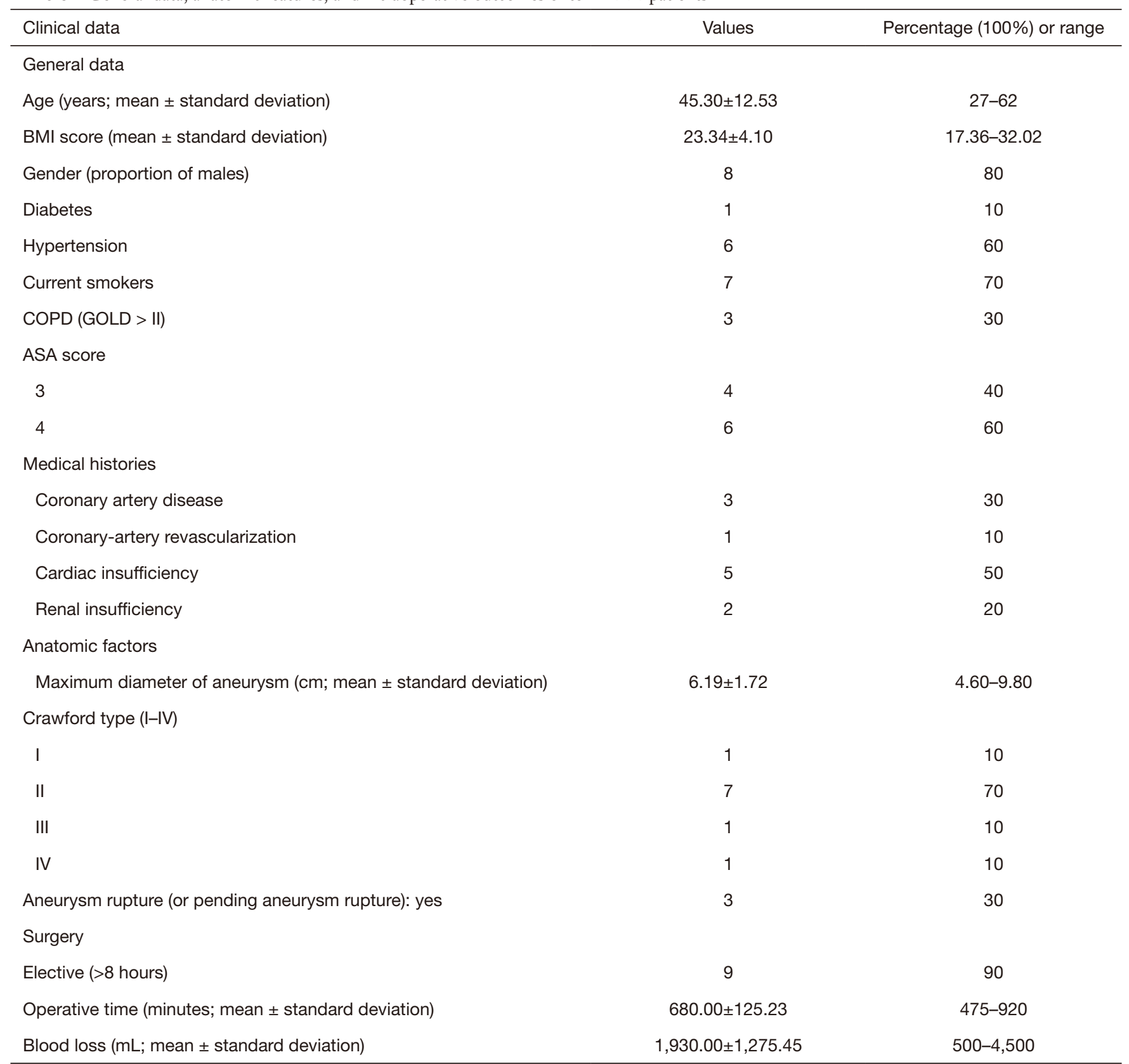

TAAA, thoracoabdominal aortic aneurysm; BMI, body mass index; COPD, chronic obstructive pulmonary disease; ASA, American Society of Anesthesiologists.

anastomosed. First, two of the four branches were selected, and about $8 \mathrm{~cm}$ vessel of each of these two branches was cut from its distal end to the proximal end. Then, end-toend anastomosis was performed with the distal end of the artificial four-branch trunk to form two leg branches using the two cut-off vessels. A bifurcation was formed at the distal end, which was then anastomosed with the bilateral iliac arteries (Figure 1).

Routine preoperative placement of cerebrospinal fluid (CSF) drainage tube, measurement of arterial pressure at the radial and dorsalis pedis arteries, and placement of a central venous catheter

Tracheal intubation was performed under general 


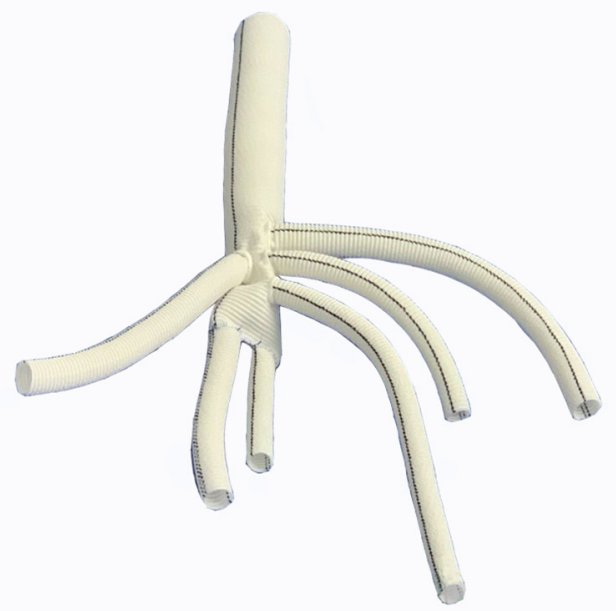

Figure 1 Modification of artificial vessels for abdominal aorta and its branches. Two of the four branches are cut down from the distal end and end-to-end anastomosis is performed with the distal ends of the vascular trunk to form two bifurcating leg branches.

anesthesia. A blood recovery system was routinely used, and hemodynamic monitoring was performed. An extracorporeal circulation system was prepared for use. An abdominal midline incision was made to expose the infrarenal abdominal aorta and bilateral iliac arteries first, followed by the exposure of the main abdominal trunk (or splenic artery), the main trunk of the superior mesenteric artery (or the proximal end of the main trunk of the superior mesenteric artery branches), and the roots of both renal arteries affected by the aneurysms.

\section{Adoption of a novel debranching technique (visceral artery reconstruction)}

The new technique quite differed from the previous vascular bypass surgery. The proximal end of the infrarenal segment of the abdominal aorta and the distal end of the bilateral common iliac arteries were mobilized first, and then both proximal and distal vascular segments of the abdominal aortic aneurysm were blocked. After the aneurysm wall incised, the proximal and distal vessels of the blocked aneurysm were transected and the stumps were severed. End-to-end anastomosis was performed between the proximal end of the artificial four-branch trunk and the infrarenal stump of the abdominal aorta, whereas end to end anastomosis was performed between the distal ends (two leg branches) of the modified artificial four-branch trunk and the bilateral common iliac artery stumps respectively.

\section{Performing anastomoses with visceral arteries}

Two artificial vessel branches of the artificial four-branch trunk were selected to perform end-lateral anastomosis with bilateral renal arteries.

One artificial vessel branch of the artificial four-branch trunk was then selected to perform end-lateral anastomosis with the superior mesenteric artery or a branch of the superior mesenteric artery posterior and inferior to the pancreas. The end-lateral anastomosis helps the matching of the caliber between the artificial vessel and the visceral vessels and also facilitates the fixation of the anastomosis at one side; as a result, the stability of the anastomosis will not be affected by the intestinal or visceral activities, and the operation becomes easier. The roots of the original visceral arteries were ligated to block the regurgitant blood flow (in celiac trunk, superior mesenteric artery, and bilateral renal arteries) into the aneurysm to prevent type II endoleak.

The last artificial vessel branch of the artificial fourbranch trunk was temporarily not anastomosed with the celiac trunk, instead, it was still clamped for subsequent endovascular surgery. If necessary, an inflow channel for extracorporeal circulation can be established via this branch vessel, and blood can be returned to the body in time; in addition, rapid infusion access can be established to ensure hemodynamic stability (Figure 2).

\section{Completion of the endovascular intervention using a new technique for thoracoabdominal trunk stenting}

The pending branch of the artificial four-branch trunk was used as the access for the endovascular intervention, and intraoperative angiography confirmed that the anastomosis between the artificial vessel and the visceral artery was patent. The stent specifications (brand, type, and size) and the proximal and distal anchorage zones were determined according to the anatomical characteristics of the aneurysms. Theoretically, the distal anchorage zone could be laid as far as immediately above the first branch of the artificial four-branch trunk. Endovascular repair of TAAA was completed using the covered stent(s) (Figure 3).

\section{Performing anastomosis with the last visceral artery}

The last branch of the artificial four-branch trunk crossed the transverse colonic mesentery along the root of the mesentery of the small intestine, forming an end-lateral anastomosis above the pancreas with the abdominal trunk or splenic artery (which is a major branch of abdominal trunk) (Figure 4). 

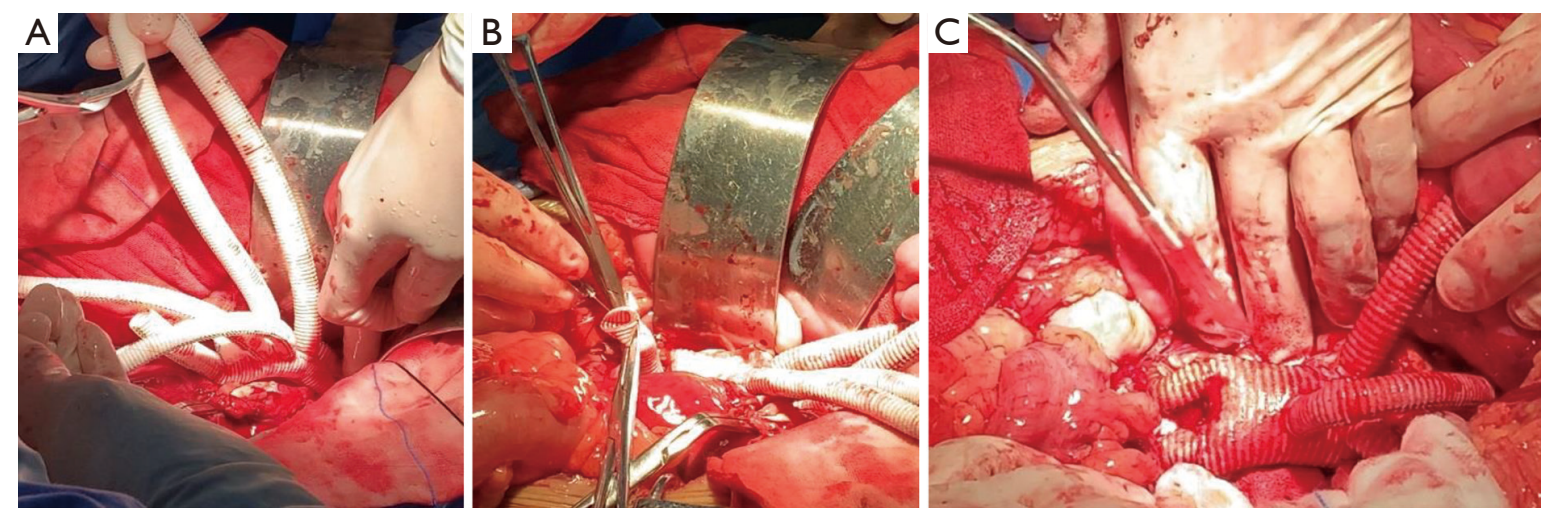

Figure 2 Visceral debranching. (A) End-to-end anastomosis is performed between the proximal end of the artificial four-branch trunk and the infrarenal stump of the abdominal aorta; (B) two leg branches of the anastomosed distal end of the artificial four-branch trunk are separately anastomosed with the stumps of bilateral common iliac arteries; and (C) anastomosis with each visceral artery is performed sequentially.
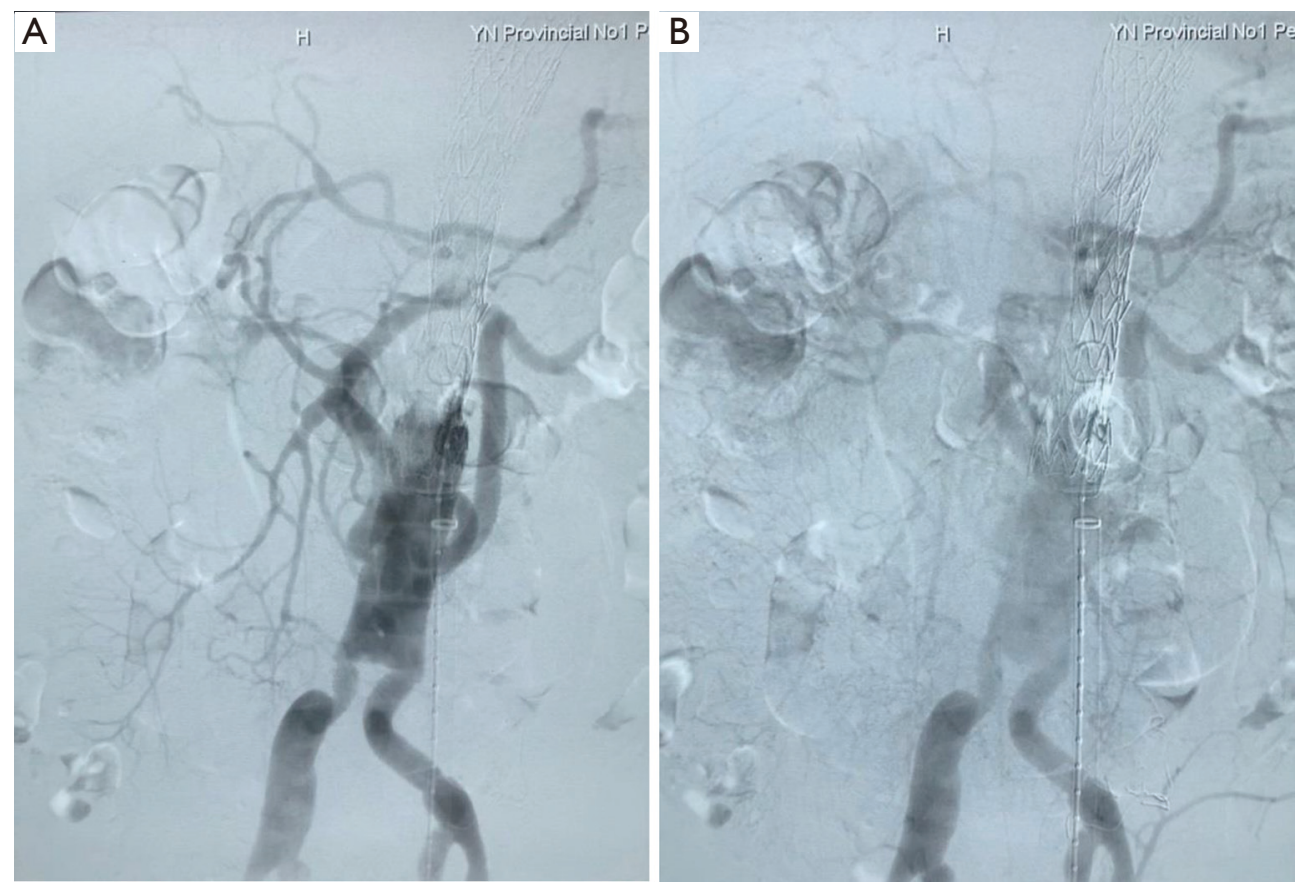

Figure 3 Endovascular intervention partially confirms the patency of the anastomoses. (A) The pending branch of the artificial four-branch trunk was used as the access for the endovascular surgery and angiography confirmed the superior mesenteric artery and bilateral iliac arteries were patent; (B) angiography showed both renal arteries were patent.

\section{Collection of key indicators}

Intraoperative key indicators including total operative time, time spent in each stage of the surgery, time for blocking abdominal aorta and each visceral vessel, blood loss, intraoperative endoleaks, patency of reconstructed vessels, and intraoperative survival were collected. Postoperative data at 30 days was directly collected from inpatients; for patients who had been discharged, data was collected via follow-up visits or telephone calls. The main data points included days of intensive care unit (ICU) stay, survival, cause of death, serious and general complications, 


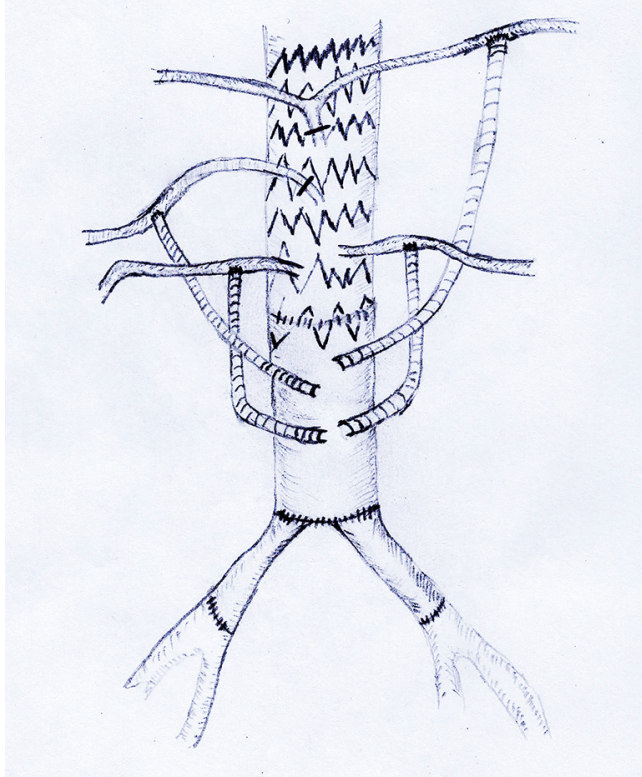

Figure 4 A pattern diagram of an anatomy-conforming hybrid surgery for TAAA. TAAA, thoracoabdominal aortic aneurysm.

functioning of all vital organs, feeding and defecation/ urination, sensorimotor status, CT angiography (CTA) findings, and adverse events. CTA was mainly used to evaluate the patency of stents, artificial blood vessels, and reconstructed visceral branches, as well as endoleaks.

\section{Statistical analysis}

Statistical analysis was performed using SPSS 19.0 software package. The count data are presented as frequencies and percentages. Normally distributed measurement data are described using means \pm standard deviations.

\section{Results}

\section{Intraoperative outcomes}

All 10 patients underwent the surgery successfully. Of these 10 patients, one patient with type IV TAAA underwent emergency hybrid surgery because of a ruptured aneurysm and already being hemodynamically unstable at the time of emergency hospitalization. The other nine cases underwent elective surgery. The operation time was $680 \pm 125 \mathrm{~min}$ (range, 475-920 $\mathrm{min}$ ), the intraoperative time for fixing the artificial vessel was $29 \pm 4 \mathrm{~min}$ (range, $23-35 \mathrm{~min}$ ), the endovascular intervention time was $28 \pm 7 \mathrm{~min}$ (range, 20-40 $\mathrm{min}$ ), and the mean intraoperative blood loss was
$1,930.00 \pm 1,275.45 \mathrm{~mL}$ (range, $500-4,500 \mathrm{~mL}$ ). The abdominal aortic cross-clamping time (the time required from the completion of the anastomoses of the infrarenal stump of abdominal aorta and the bilateral common iliac artery stumps with artificial vessel to declamping the aorta) was $54 \pm 10 \mathrm{~min}$ (range, $40-70 \mathrm{~min}$ ), the right renal artery cross-clamping time was $16 \pm 4 \mathrm{~min}$ (range, $12-25 \mathrm{~min}$ ), the left renal artery cross-clamping time was $28 \pm 7 \mathrm{~min}$ (range, 14-28 $\mathrm{min}$ ), the superior mesenteric artery cross-clamping time was $14 \pm 3 \mathrm{~min}$ (range, 10-20 $\mathrm{min}$ ), and the abdominal trunk artery (or its branches) cross-clamping time was $15 \pm 3$ min (range, 10-20 min). No endoleak occurred after intraoperative stent implantation and the blood supply of each reconstructed visceral artery was good. No intraoperative death was noted (Table 1).

\section{0-day postoperative outcomes}

All of the 10 patients have been followed up and their postoperative outcomes in 30 days were analyzed. The duration of the postoperative hospital stay was $25.0 \pm 8.0$ days (range, $12-37$ days). ICU stay was $6.9 \pm 4.6$ days (range, $4-18$ days). One patient died from non-aortic causes (sepsis/infectious toxic shock) 24 days after surgery, yielding a case-fatality rate of $10.0 \%(1 / 10)$. The reoperation rate was $10 \%(1 / 10)$ within 30 days as one patient suffered from left lower extremity ischemia on postoperative day 3 and required emergency left iliac artery stenting. Two patients $(2 / 10)$ experienced serious complications (fatal or serious complications affecting organ function and quality of life) within 30 days postoperatively, including multi-organ failure due to infection in one patient who was ultimately died as mentioned above and lower limb ischemia (mentioned above) in another patient who went on to develop permanent paraplegia. The newlyonset complications included cardiac insufficiency $(n=4)$, respiratory failure $(n=3)$, pulmonary infection $(n=6)$, chylous leakage $(n=7)$, abnormal liver function $(n=2)$, renal insufficiency $(n=5)$, and intestinal obstruction $(n=1)$.

Considering that cardiopulmonary insufficiency was related to intraoperative hemodynamic fluctuations and hepatic and renal insufficiencies were more likely to be associated with visceral arterial blockage, we offered symptomatic support for organ insufficiencies in the postoperative period. Pulmonary infections were treated with antimicrobials and enhanced expectoration of sputum, and patients were encouraged to become ambulatory early. Chylous leakage was treated with total parenteral nutrition 
Table 2 30-day postoperative survival rate, major causes of death, and complications

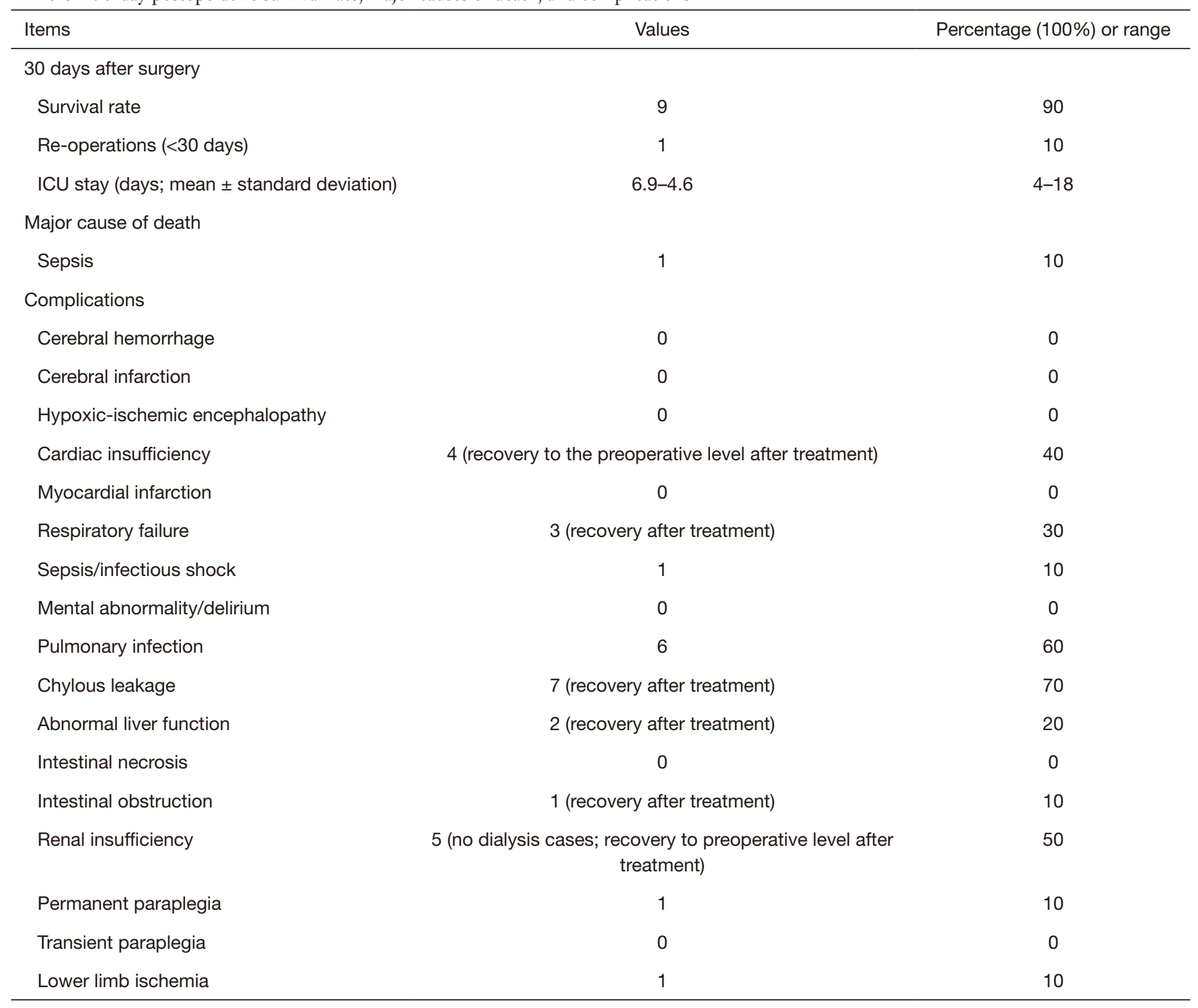

ICU, intensive care unit.

and low-fat diet. The above general complications were improved and recovered after symptomatic treatment, thus representing only a transient manifestation. No endoleak after stent implantation was noted and the blood supply of each reconstructed visceral artery was good (Table 2).

\section{Discussion}

A variety of surgical options represented by traditional open surgery, complete endovascular treatment, and hybrid surgery have been applied in the treatment of TAAAs. However, their effectiveness, shortcomings and modifications have long been controversial. With the advances in treatment concepts, surgical levels and endovascular devices, the treatment of TAAA has been thriving and flourishing.

The value of hybrid surgery in treating TAAA has increasingly been recognized as it combines the advantages of both open surgery and endovascular techniques. (Search on PubMed using the key word "thoracoabdominal aortic aneurysm, hybrid" yielded an average of 103 articles annually in the past decade, showing a rising trend since 2005). However, it has also been questioned that the visceral vessel reconstruction technique (including retrograde 
and prograde bypass for visceral debranching) used in the current hybrid surgery is still performed via a non-anatomic route. In general, the prograde bypass is only suitable for type IV TAAA. In contrast, the retrograde approach to visceral debranching usually uses an inverted bifurcated artificial vessel as a bypass graft, with the abdominal aorta or iliac artery as the inflow tract, which is retrogradely anastomosed to the artificial vessel trunk, followed by the anastomosis between each visceral artery and the artificial vessel branch to enable the retrograde perfusion of the visceral artery (4).

To address this problem, we developed a new hybrid surgery involving infrarenal aortic replacement and stent implantation, during which the HEMASHIELD Platinum artificial vessel, which is commonly used in open thoracic surgery, is applied for vascular reconstruction. The main technical advantages of this hybrid surgery include: (I) it maximizes the restoration of normal hemodynamics, rendering it more consistent with the original anatomical and physiological features, and in addition, the branches offer more adequate organ perfusion; (II) it avoids a second intervention for lesions in the inflow tract (roughly normal abdominal aorta or iliac artery) due to compensatory expansion of the vessel after a bypass surgery; and (III) it minimizes the incidence of complications such as endoleaks after endovascular stent-graft exclusion of the diseased segment of the abdominal aorta.

In the literature, one article reported a case-fatality rate of $11.8 \%$ in 17 patients after hybrid surgery (3). In another study that included 52 patients, the perioperative mortality rate for hybrid surgery was $8.6-13.5 \%$ (7). In a study comparing hybrid surgery with a completely open or endovascular approach, the overall 30-day case-fatality rate was $13 \%$ and the overall complication incidence was $33 \%$ in 40 patients who received hybrid surgery (8). In another study that included 10 patients undergoing hybrid surgery, three patients $(30 \%)$ required postoperative dialysis, but none of them required it permanently; one patient (10\%) experienced paraplegia (which was transient in other patients and improved by cerebrospinal fluid drainage) (9).

Our center has currently completed anatomyconforming hybrid surgery for 10 TAAA patients. The 30 -day survival rate reached $90 \%$. Two patients $(20 \%)$ had serious complications, including severe infection in one case $(10 \%)$ and permanent paraplegia in another case $(10 \%)$. The general complications included insufficiency or dysfunction of one or several organs, which recovered well after symptomatic treatment. Generally, the key indicators such as survival and complication rates of patients in the perioperative period were basically the same as in patients undergoing conventional hybrid surgery. However, the mid- and long-term outcomes (especially survival, function of the perfused organs by the vessel branches, incidence and extent of distal iliac artery dilatation, and incidence and type of endoleaks in the abdominal aorta) and their advantages (if any) over the traditional hybrid repair still warrant further investigations.

Of the 10 patients, one fatal case was a 47 -year-old male who was treated with endovascular exclusion for aortic type B dissection on May 5, 2014 and admitted to our center due to pending Type II TAAA rupture on Sep 9, 2019. This patient received the current hybrid repair subsequently and the entire procedure was smooth. During and after the surgery, the multiple organ support therapy and prophylactic anti-infection were regularly applied. However, the patient gradually appeared systematic infection and on 15 th day after surgery, multiple susceptibility tests indicated multi-drug-resistant bacteria in his blood. After a period of combined anti-infective treatment, the bacteria in patient's blood became resistant to almost all regular antibiotics and led to more severe infection. The patient died on 24th day after surgery followed by multisystem organ failure involving the liver, kidneys, coagulation system, and heart. The reasons of his death are not directly correlated with the surgery itself but with the complicated sepsis secondary to the surgery. To reduce the operation time, standardize antibiotic use and strengthen infection control could be viable solutions to severe infection after surgery.

A 55-year-old male patient with severe complications developed permanent paraplegia after surgery. An indwelling cerebrospinal fluid drainage tube is routinely applied after our hybrid surgery to reduce the incidence of postoperative paraplegia. The duration is about three days, and the daily drainage volume is $80-120 \mathrm{~mL}$. Patients are closely monitored for neuromotor symptoms and signs and asepsis rules are strictly observed to avoid neurological infections. The incidence of paraplegia has been reported to be $7-11 \%$ (9). Quiñones-Baldrich et al. reviewed 16 aortic dissection patients with TAAAs and/or malperfusion and found that in nine patients $(56 \%)$ cerebrospinal fluid drainage tubes were used. Carotid-subclavian bypass was required in patients with coverage of subclavian artery (six patients, 38\%) (10).

What should be paid more attention is perioperative comprehensive management, which is crucial to guarantee better recovery for TAAA hybrid patients. In severe 
cases, TAAAs are combined with rupture or abnormal perfusion of organs, indicating the disease has progressed and become critical and even life-threatening. Therefore, careful preoperative planning, intraoperative maneuvers, and postoperative management are particularly important $(11,12)$. We should focus on sustaining the functions of cardiovascular, respiratory, neural, renal and coagulation system, relying on professional group construction, special intensive care unit support, and nursing quality improvement.

In conclusion, the hybrid surgery transforms type II/III TAAA into type IV for repair, thus lowering the open surgical plane and providing more anchorage areas. In our center, the anatomy-conforming hybrid surgery that combines infrarenal aortic replacement with stenting, restores hemodynamics to its original state to the greatest extent possible. However, our current study was limited by its small number of cases and short follow-up period. Studies with more adequate intermediate- and long-term data are warranted to further evaluate the effectiveness of this innovative surgical approach, further improve patient prognosis and reduce complications. The application of staged surgery, the value of cerebrospinal fluid drainage in reducing ischemic damage to the spinal cord and the role of extracorporeal circulation techniques in this procedure should also be considered and will be explored in our future studies.

\section{Acknowledgments}

Funding: Supported by Chen Zhong Expert Workstation Fund of Yunnan Province (2005AF150018).

\section{Footnote}

Reporting Checklist: The authors have completed the STROBE reporting checklist. Available at https://dx.doi. org/10.21037/apm-21-1569

Data Sharing Statement: Available at https://dx.doi. org/10.21037/apm-21-1569

Conflicts of Interest: All authors have completed the ICMJE uniform disclosure form (available at http://dx.doi.org/ apm-21-1569). The authors have no conflicts of interest to declare.

Ethical Statement: The authors are accountable for all aspects of the work in ensuring that questions related to the accuracy or integrity of any part of the work are appropriately investigated and resolved. All the patients and their families signed informed consent forms. The study was approved by the Research Ethics Sub-Committee of the Medical Ethics Committee of the First People's Hospital of Yunnan Province (approval number: KHLL2020-KY049). All procedures performed in this study involving human participants were in accordance with the Declaration of Helsinki (as revised in 2013).

Open Access Statement: This is an Open Access article distributed in accordance with the Creative Commons Attribution-NonCommercial-NoDerivs 4.0 International License (CC BY-NC-ND 4.0), which permits the noncommercial replication and distribution of the article with the strict proviso that no changes or edits are made and the original work is properly cited (including links to both the formal publication through the relevant DOI and the license). See: https://creativecommons.org/licenses/by-nc-nd/4.0/.

\section{References}

1. Kalder J, Kotelis D, Jacobs MJ. Thoracoabdominal aortic aneurysm. Chirurg 2016;87:797-810.

2. Quiñones-Baldrich WJ, Panetta TF, Vescera CL, et al. Repair of type IV thoracoabdominal aneurysm with a combined endovascular and surgical approach. J Vasc Surg 1999;30:555-60.

3. Di Marco L, Murana G, Leone A, et al. Hybrid repair of thoracoabdominal aneurysm: An alternative strategy for preventing major complications in high risk patients. Int $\mathrm{J}$ Cardiol 2018;271:31-5.

4. Yamaguchi D, Jordan WD Jr. Hybrid thoracoabdominal aortic aneurysm repair: current perspectives. Semin Vasc Surg 2012;25:203-7.

5. Rosenblum JM, Chen EP. Thoracoabdominal aortic aneurysm repair: open, endovascular, or hybrid? Gen Thorac Cardiovasc Surg 2019;67:175-9.

6. Mestres CA, Tsagakis K, Pacini D, et al. One-stage repair in complex multisegmental thoracic aneurysmal disease: results of a multicentre study. Eur J Cardiothorac Surg 2013;44:e325-31.

7. Tshomba Y, Melissano G, Logaldo D, et al. Clinical outcomes of hybrid repair for thoracoabdominal aortic aneurysms. Ann Cardiothorac Surg 2012;1:293-303.

8. Arnaoutakis DJ, Scali ST, Beck AW, et al. Comparative outcomes of open, hybrid, and fenestrated branched 
endovascular repair of extent II and III thoracoabdominal aortic aneurysms. J Vasc Surg 2020;71:1503-14.

9. Värämäki S, Suominen V, Pimenoff G, et al. Hybrid Repair of Thoracoabdominal Aortic Aneurysms Is a Durable Option for High-Risk Patients in the Endovascular Era. Vasc Endovascular Surg 2016;50:491-6.

10. Quiñones-Baldrich WJ, Saleem T, Oskowitz A. Infrarenal aortic repair with or without false lumen intentional placement of endografts for hybrid management of complex aortic dissection. J Vasc Surg 2018;68:46-54.

Cite this article as: Li Y, Xiao L, Zhang P, Zhang J, Guo S, Li R, Wei Y, Gong K. Short-term effectiveness and safety of an anatomy-conforming hybrid surgery protocol for thoracoabdominal aortic aneurysms. Ann Palliat Med 2021;10(8):8762-8771. doi: 10.21037/apm-21-1569
11. Bensley RP, Curran T, Hurks R, et al. Open repair of intact thoracoabdominal aortic aneurysms in the American College of Surgeons National Surgical Quality Improvement Program. J Vasc Surg 2013;58:894-900.

12. Chiesa R, Melissano G, Civilini E, et al. Video-atlas of hybrid thoracoabdominal aortic aneurysm repair. Ann Cardiothorac Surg 2012;1:404-5.

(English Language Editor: M. Bucci) 